\title{
Clinical trials of Cry j 1 and Cry j 2 T-cell epitope peptide-expressing rice in patients with Japanese cedar pollinosis
}

\author{
Shoji Hashimoto, ${ }^{1}$ Masatoshi Kawata, ${ }^{1}$ Nobusuke Mino, ${ }^{2}$ Satoshi Ogino, ${ }^{3}$ Noriaki Takeda, ${ }^{4}$ Kayoko Kawashima, ${ }^{5}$ \\ Hidenori Takagi, ${ }^{6}$ Yuhya Wakasa, ${ }^{6}$ Makoto Takano, ${ }^{6}$ Masaki Tanaka, ${ }^{7}$ Yuji Tohda, ${ }^{8}$ Toshio Tanaka ${ }^{9}$
}

\begin{abstract}
Background: In allergic models, administration of rice that expresses a hybrid peptide consisting of 7 major $\mathrm{T}$ cell epitopes of Cry j 1 and Cry j 2 (7Crp), suppressed allergic symptoms, IgE elevation and specific T cell response to Japanese cedar pollen.
\end{abstract}

Objectives: To evaluate the efficacy and safety of 7Crp-expressing rice in patients with Japanese cedar pollinosis

Methods: A 24-week randomized, double-blind, placebo-controlled study was performed to see the efficacy of 7Crp on allergic symptoms using scoring systems, in which 45 patients were assigned to take either $5 \mathrm{~g}, 20 \mathrm{~g}$ test rice, or placebo daily. A 96-week open study was also conducted to determine its inhibitory effect on serum IgE and T cell proliferative response for Japanese cedar pollen, in which 10 patients consumed $5 \mathrm{~g}$ test rice daily.

Results: No adverse events associated with the test rice occurred, and the intake rate was more than $96 \%$. The test rice did not show suppression of symptoms related to Japanese cedar pollinosis within 24 weeks. However, intake of $5 \mathrm{~g}$ test rice led to a significant decrease in $\mathrm{T}$ cell response to Japanese cedar pollen during and after the second disperse season in a 96-week open trial, whereas the specific IgE titer remained unchanged.

Conclusion: Tolerability and safety of 7Crp-expressing rice was accepted. Daily intake of up to 20 g transgenic rice did not provide beneficial effects on Japanese cedar pollinosis within 24 weeks, however, continuous intake of $5 \mathrm{~g}$ rice might reduce allergen specific $\mathrm{T}$ cell response.

Key words: Cry j 1 and Cry j 2, genetically modified rice, Japanese cedar pollinosis, oral allergen immunotherapy, T cell epitope peptide.

\section{Citation:}

Hashimoto, S., Kawata, M., Mino, N., Ogino, S., Takeda, N., Kawashima, K., Takagi, H., Wakasa, Y., Takano, M., Tanaka, M., Tohda, Y., Tanaka, T. (2022). Clinical trials of Cry j 1 and Cry j $2 \mathrm{~T}$-cell epitope peptide-expressing rice in patients with Japanese cedar pollinosis. Asian Pac J Allergy Immunol, 40(4), 386-392. https://doi.org/10.12932/ap-050619-0575

\section{Affiliations:}

${ }^{1}$ Department of Clinical Laboratory, Osaka Prefectural Hospital Organization Osaka Habikino Medical Center, Osaka, Japan

${ }^{2}$ Secretariat, Osaka Prefectural Hospital Organization Osaka Habikino Medical Center, Osaka, Japan

3 Osaka Health Care and Allergy Support Organization, Osaka, Japan

${ }^{4}$ Department of Otolaryngology, University of Tokushima School of

Medicine, Tokushima, Japan
${ }^{5}$ Department of Otorhinolaryngology, Osaka Prefectural Hospital Organization Osaka Habikino Medical Center, Osaka, Japan

${ }^{6}$ Institute of Agrobiological Sciences, National Agriculture and Food Research Organization, Ibaraki, Japan

Masaki Clinic, Osaka, Japan

${ }^{8}$ Department of Respiratory Medicine and Allergology, Kinki University, Osaka, Japan

${ }^{9}$ Medical Affairs Bureau, Osaka Prefectural Hospital Organization Osaka Habikino Medical Center, Osaka, Japan

\section{Corresponding author:}

Toshio Tanaka

Medical Affairs Bureau, Osaka Prefectural Hospital Organization Osaka Habikino Hospital,

3-7-1 Habikino, Habikino City, Osaka 583-8588, Japan

E-mail: ttanak@ra.opho.jp 


\section{Introduction}

The prevalence of allergic diseases including asthma, atopic eczema, allergic rhinitis and food allergy has increased worldwide during the past two to three decades. ${ }^{1}$ In Japan, the prevalence rate of Japanese cedar pollinosis (JCP) dramatically increased from $16.2 \%$ in 1998 to $26.5 \%$ in 2008 according to a nationwide survey. ${ }^{2}$ A recent epidemiologic study reported that $78.8 \%$ of Japanese young adults are now sensitized with Japanese cedar pollen, and the prevalence of JCP is $41.2 \%$ based on subjective symptoms and $22.2 \%$ based on physician diagnosis of the population. ${ }^{3}$ The disease severely affects the quality of life (QOL), leading to loss of social activity of patients and inflicts an economic burden on the society. ${ }^{4}$ Thus, preventive strategies and innovative therapeutic approaches for the pollinosis need to be developed. Subcutaneous or sublingual allergen-specific immunotherapy (AIT) using Japanese cedar pollen extract has proved to be effective with a tolerable safety profile, and is used for the treatment of patients. $^{4-5}$ However, there are still several concerns regarding the present methodology of AIT, which need to be addressed. ${ }^{6-7}$ First, it is efficacious for hyposensitization, however, not so for complete desensitization to allergens. Second, the therapy, in some cases, induces immediate allergic reaction, as the extract stimulates mast cells or basophils in an IgE-dependent manner. Third, it takes from several months to years to show its effect; thus, substantial numbers of patients abruptly terminate the therapy. Moreover, the precise mechanism(s) underlying its efficacy have not been clarified.

Transgenic rice, or genetically modified rice that expresses 25-62 $\mu \mathrm{g}$ /grain of a hybrid peptide including 7 major $\mathrm{T}$ cell epitopes of Cry j 1 and Cry j 2 (7Crp), has several potential beneficial characteristics which might help to overcome the aforementioned difficulties. First, the peptide cannot bind to Japanese cedar pollen-specific IgE, therefore, theoretically, does not induce mast cell activation. ${ }^{8}$ However, it can facilitate the cellular immune response towards tolerance. ${ }^{9}$ Second, the peptide is localized in the endosperm of rice and reaches the ileum and jejunum without digestion by gastrointestinal enzymes. Thus, it is likely to be absorbed by gut-associated lymphatic tissue. ${ }^{9,10}$ Third, as rice is the staple food in Japan, it is convenient for patients to continuously consume the transgenic rice. Thus, we evaluated the efficacy, safety and tolerability of the transgenic rice in patients with JCP.

\section{Methods \\ Participants}

A total of 79 adult patients, who live or work in Osaka Prefecture and have had allergic symptoms related to JCP at least over the past 2 years, were assessed for eligibility of the study. Nine patients showed negative blood IgE (less than class 2 titer) specific for Japanese cedar pollen and then excluded. Moreover, 15 patients were excluded on the basis of exclusion criteria set for patients: 1. Positive IgE for rice, 2. More than class 5 titer of IgE for Japanese cypress pollen,
3. More than class 5 titer of $\operatorname{IgE}$ for house dust mites, 4. Individuals with pets (dogs or cats) at home with more than class 5 titer of IgE for the corresponding pet, 5 . Individuals receiving AIT or those who will receive AIT during the study, and 6. Individuals with systemic diseases. Exclusion criteria items 2-4 were set to minimize allergic symptoms due to other allergens. Then, a total of 55 patients were enrolled for the study. Studies were designed in accordance with the Helsinki declaration and approved by the Ethics Committee of the Osaka Habikino Medical Center (approved number: 787-7). All participants provided written informed consent. The registration numbers of the studies in University hospital Medical Information Network (UMIN) are UMIN-000024699 and UMIN-000024700.

\section{Generation of transgenic rice}

Cry j 1 and Cry j 2 are major allergens in Japanese cedar pollen and there are 3 dominant $\mathrm{T}$ cell determinants (residues 212-224, 235-247 and 312-330) in Cry j 1, and 4 (residues 77 89, 96-107, 192-204 and 356-367) in Cry j 2.11 We developed a hybrid peptide consisting of 96 amino acids with a molecular weight of $10.6 \mathrm{kDa}$, in which these $7 \mathrm{~T}$ cell determinants were arranged in tandem, using an Escherichia coli expression vector. ${ }^{11}$ 7Crp could induce a $\mathrm{T}$ cell proliferative response in 44 out of 48 (92\%) patients with JCP. We then prepared a construct including the 7Crp gene under the rice major seed storage protein glutein $2.3-\mathrm{kb} G l u B-1$ promoter, and transferred it into the rice genome (Oryza sativa cv. Kita-ake) using Agrobacterium tumefaciens-mediated transformation. ${ }^{10,12}$ The amount of 7Crp in the transgenic rice used for this study was 7.5-8.6 $\mu \mathrm{g}$ per $5 \mathrm{mg}$ rice, measured using SDS-PAGE, followed by Western blotting using an anti-7Crp antibody, as described previously. ${ }^{11}$ The packed rice was prepared and taken once daily after warmed in the microwave.

\section{Design of the study}

We conducted two studies. One is a randomized, doubleblind, placebo-controlled study, in which 45 patients with JCP were assigned to receive either $5 \mathrm{~g}$ transgenic rice including 8 mg 7Crp, mixed with $45 \mathrm{~g}$ placebo rice, $20 \mathrm{~g}$ transgenic rice including $32 \mathrm{mg}$ 7Crp, mixed with $30 \mathrm{~g}$ placebo rice, or 50 g placebo rice once a day for 24 weeks. Randomization was stratified by gender and the titer of specific IgE for Japanese cedar pollen by using random permuted block sizes of 4 or 6. The second is an open study, in which 10 patients with JCP were designated to take $5 \mathrm{~g}$ transgenic rice, mixed with $45 \mathrm{~g}$ placebo rice for 96 weeks. Both studies commenced on November 6, 2016. The primary endpoint of study 1 was based on a change in the subjective symptom score, whereas secondary endpoints include changes in the QOL score, medication score and specific IgE titer for Japanese cedar pollen, and the safety profile of the method. In study 2 the primary endpoint was based on a change in specific IgE titer for Japanese cedar pollen and secondary endpoints include a change in the $\mathrm{T}$ cell proliferative response to Japanese cedar pollen and the safety profile of the method. 
In 2017, the regional records for dispersal of Japanese cedar pollen in Osaka Prefecture showed that the onset, peak and end of cedar pollen release occurred on February 19, March 20 and April 16, respectively. The peak and total amounts of its release were 111 grains/day and 842 grains, respectively. In 2018, the release of Japanese cedar pollen began on February 23, and ended on April 8, with the total amounts being 1441, with the peak on March 7 (106 grains/ day).

\section{Outcome measures}

Severity of symptoms was determined using a scoring system based on slightly modified method by Baba et al. ${ }^{13}$ Participants were asked to record symptom scores and disturbance of activities of daily living (ADL) every day. Symptoms were evaluated according to the following scale: sneezing and rhinorrhea $(0=$ none, $1=1-5$ episodes, $2=6-10$, $3=11-20$, and $4=>20$ episodes $)$, nasal obstruction $(0=$ none, 1 = little without oral breathing, 2 = mild with sometimes oral breathing, 3 = severe with often oral breathing, $4=$ complete obstruction almost all day), dysgeusia $(0=$ none, $1=$ mild, $2=$ moderate, $3=$ severe $)$, disturbance of ADL $(0$ = none, $1=$ mild, $2=$ between 1 and $2,3=$ moderate, $4=$ severe), ocular itching $(0=$ none, $1=$ mild, $2=$ moderate, $3=$ severe) and lacrimation ( $0=$ none, $1=$ mild, $2=$ moderate, 3 $=$ severe). The total symptom score was calculated as a sum of these 7 indices.

Participants were allowed to take oral antiallergic drugs and to use ocular drops and nasal sprays. The consumption of these drugs was recorded and evaluated by the medication score according to the Japanese Society of Allergology with the following scale: $0=$ none, $1=$ oral antihistamines, oral histamine release inhibitors, nasal sprays excluding focal steroids and ocular drops excluding focal steroids, $2=$ focal administration of steroids, 3 = oral administration of steroids. ${ }^{13}$ The symptom score and medication score were recorded daily from February 1 to May 2 in 2017.

The degree of disturbance of QOL was assessed using the Japanese Allergic Rhinitis QOL Questionnaire (JRQLQ No.1). ${ }^{14}$ It comprises 17 questions which include details like rhinorrhea, sneezing, nasal obstruction, nasal and ocular itching, lacrimation, general fatigue, irritability, depression and difficulties with daily activities such as working, housekeeping, studying, reading, sports, going outdoors, sleeping and having conversations. Participants were asked to record the QOL score every 2 weeks from February 1 to April 16 in 2017.

\section{Blood sampling}

Blood samples were collected before the study and then after every 12 weeks until the end of the study. Serum specific IgE, IgG and IgG4 for Japanese cedar pollen, cypress, house dust mite, cats and dogs were measured using the AlaSTAT3g method (SIEMENS, range; 0.1 500 IU/ml). In study 1, these levels were also monitored at 24 weeks after the intervention period. In study 2 , in addition to the measurement of these IgE levels, the allergen-specific lymphocyte stimulation test was performed before the study and then after every 12 weeks. Briefly, peripheral blood mononuclear cells $\left(2 \times 10^{5}\right.$ cells/well $)$ were cultured with or without $2.5 \mu \mathrm{g} / \mathrm{mL}$ purified Japanese cedar pollen basic protein (Hayashibara Co., LTD, Okayama, Japan), $1.25 \mu \mathrm{g} / \mathrm{mL}$ purified protein derivative (Japan BCG Laboratory, Tokyo, Japan), or $10 \mu \mathrm{g} / \mathrm{mL}$ phytohemagglutin for 3 days, then ${ }^{3} \mathrm{H}$-thymidine was added and cells were further cultured for 17 hours, and ${ }^{3} \mathrm{H}$-thymidine incorporation was measured using a liquid scintillation counter. Stimulation index (SI) was calculated as (mean cpm of allergen-added wells) $/$ (mean cpm of unstimulated wells).

\section{Statistical analysis}

Quantitative data are presented as means \pm SEM, unless indicated otherwise. The significance of the difference between groups in study 1 was evaluated using the Mann-Whitney $U$ test, and the significance of the changes in values from the baseline to 96 weeks in study 2 was evaluated using the paired Student's $t$ test. A value of $P<0.05$ was considered as statistically significant.

\section{Results \\ Characteristics of patients}

Characteristic features of enrolled patients are presented in Table 1. In study 1 baseline characteristics of patients were similar and there were no significant differences in age, disease duration, sex distribution, and the titer of specific IgE for Japanese cedar pollen and cypress pollen among the groups.

\section{Table 1. Characteristics of enrolled patients}

\begin{tabular}{|c|c|c|c|c|c|}
\hline & \multicolumn{4}{|c|}{ Study 1} & \multirow{2}{*}{$\begin{array}{c}\text { Study } 2 \\
\mathbf{5} \text { g group }\end{array}$} \\
\hline & 5 g group & 20 g group & $\begin{array}{c}\text { Placebo } \\
\text { group }\end{array}$ & $\begin{array}{l}\text { p-value } \\
\text { (ANOVA) }\end{array}$ & \\
\hline Number of entries & 15 & 15 & 15 & & 10 \\
\hline Age & $38.8 \pm 14.0^{\dagger}$ & $41.4 \pm 11.9$ & $40.5 \pm 11.9$ & NS & $43.7 \pm 12.0$ \\
\hline Duration of disease (year) & $21.3 \pm 14.0$ & $19.4 \pm 8.8$ & $15.0 \pm 7.6$ & NS & $19.9 \pm 11.1$ \\
\hline Gender (male/female) & $9 / 6$ & $9 / 6$ & $10 / 5$ & NS & $2 / 8$ \\
\hline IgE for Japanese cedar pollen (IU/ml) & $19.7 \pm 18.2$ & $20.3 \pm 18.2$ & $18.3 \pm 16.4$ & NS & $16.0 \pm 12.2$ \\
\hline IgE for cypress pollen (IU/ml) & $8.7 \pm 7.3$ & $10.0 \pm 12.0$ & $8.4 \pm 8.0$ & NS & $6.9 \pm 6.7$ \\
\hline
\end{tabular}

†S.D., NS: not significant 
Effects of the transgenic rice on symptoms related to JCP

The onset of Japanese cedar pollen release occurred on February 19 and the peak on March 20 in 2017. As shown in Figure 1, the symptoms related to rhinitis and conjunctivitis were conspicuous before dispersal; however, they aggravated proportionally with the amounts of Japanese cedar pollen released. The average symptom score of the placebo group before and during the pollen season was marginally lower than that of the $5 \mathrm{~g}$ and $20 \mathrm{~g}$ intake group but the difference was not statistically significant. Moreover, there were no significant differences in changes in the QOL and the medication score among the three groups, indicating that
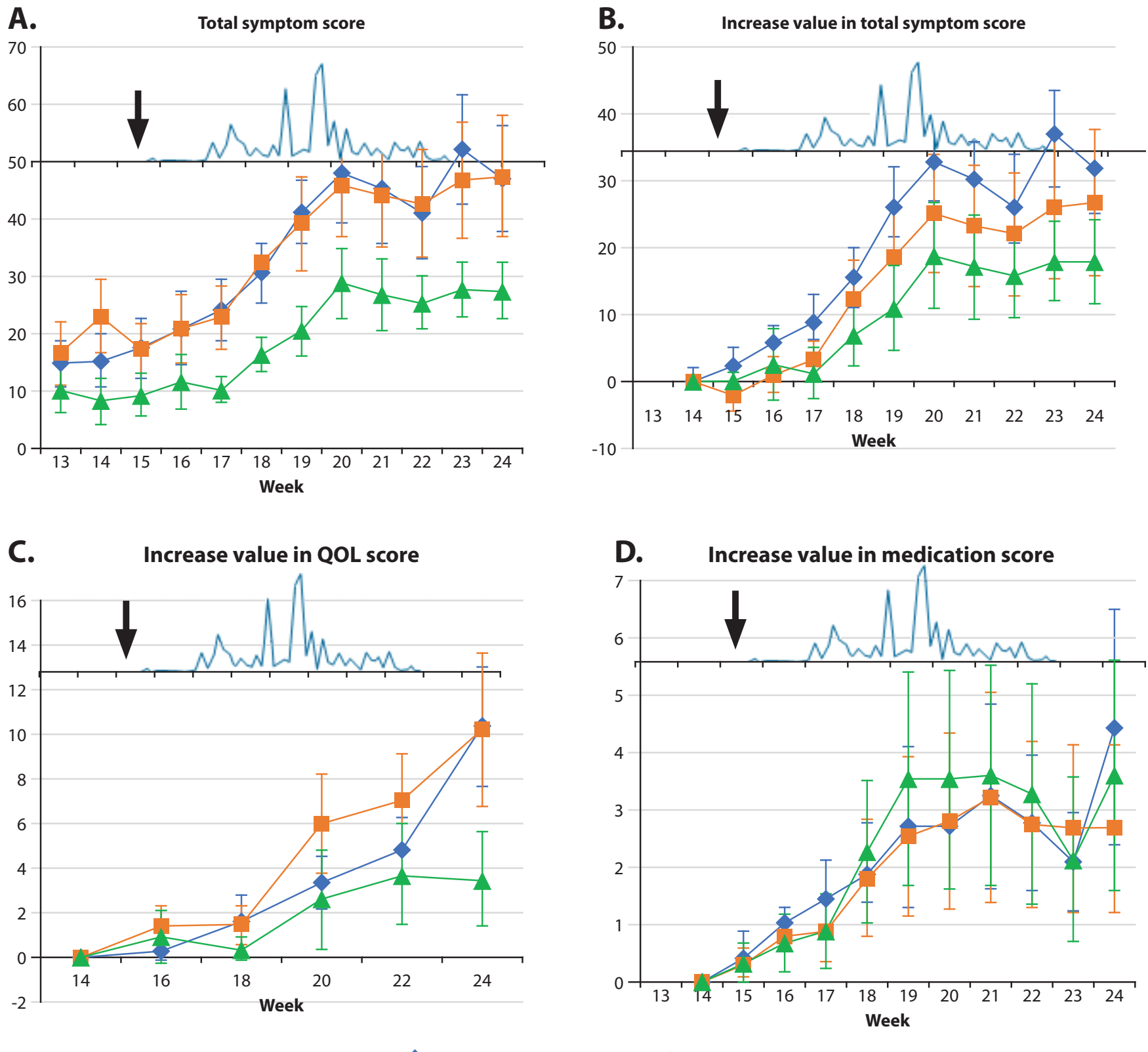

24-week intake of up to $20 \mathrm{~g} 7 \mathrm{Crp}$-expressing rice did not show ameliorative effects on allergic symptoms of patients with JCP.

\section{Effects of the transgenic rice on immunological parameters}

The change in the serum IgE titer for Japanese cedar pollen in study 1 is shown in Figure 2. After the pollen season, the titer increased in all three groups; however, there was no significant difference in the titer among the three groups. Moreover, IgG and IgG4 levels for Japanese cedar pollen were not significantly different among the groups (data not shown).

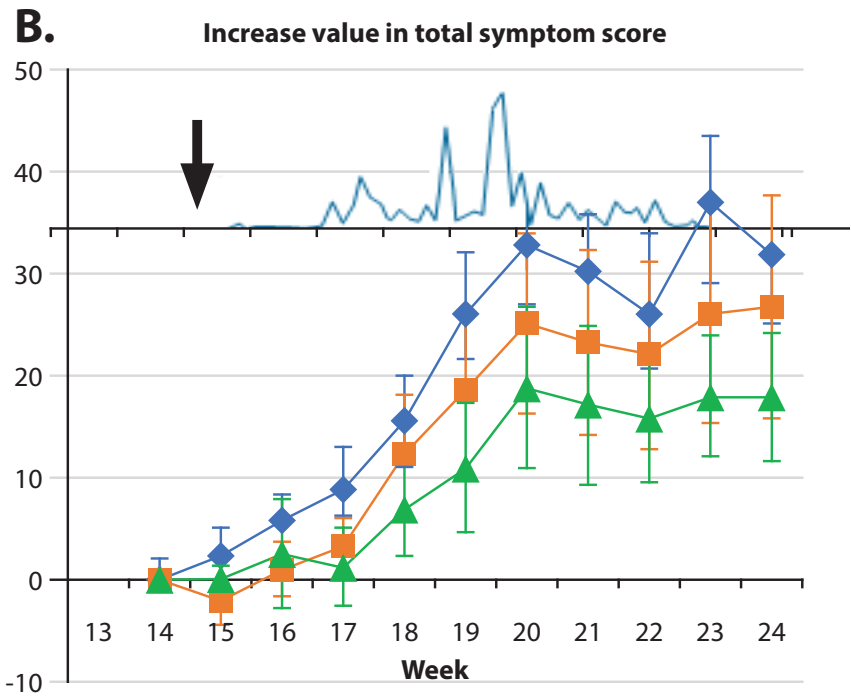

Figure 1. Changes in total symptom score, QOL score, and medication score

A. Change in total symptom score during the entire test period. B. Change in increase value in the total symptom score during the pollen season. C. Change in increase value in the QOL score during the pollen season. D. Change in increase value in the medication score during the pollen season. The increase value is shown as each value subtracted from the average value before the onset of pollen dispersal. Each value represents mean \pm SE. 


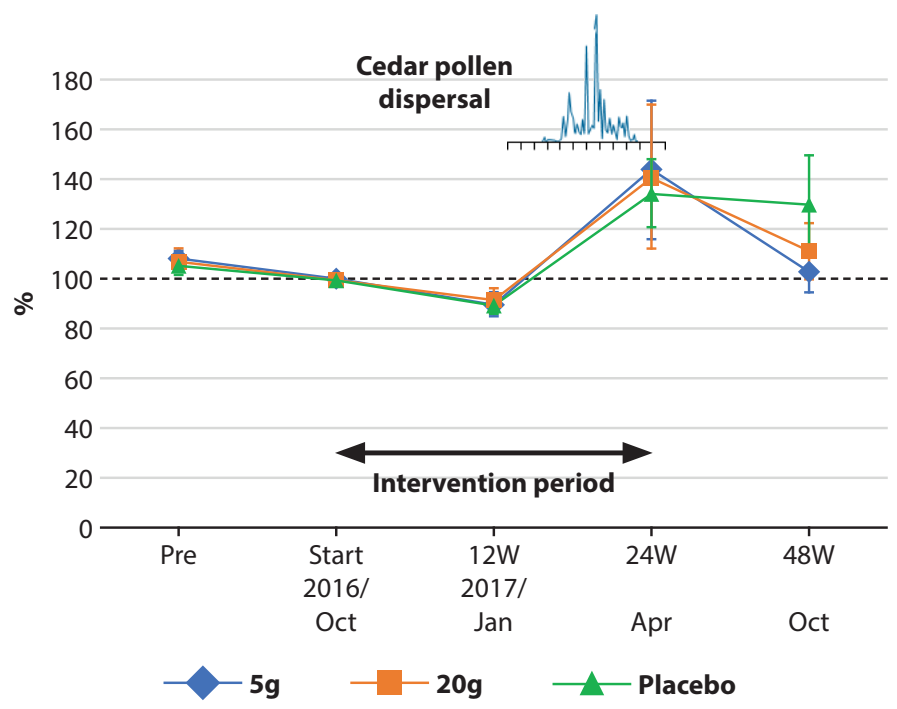

Figure 2. Change in levels of serum IgE specific to Japanese cedar pollen in study 1

Each value represents mean $\pm \mathrm{SE}$.

Study 2 examined the long-term (96 weeks) effect of the $5 \mathrm{~g}$ transgenic rice on the proliferative response of peripheral blood $\mathrm{T}$ cells to Japanese cedar pollen basic protein. The response increased during and 3 months after the pollen season in the first year (Figure 3). However, in the second season of the pollen dispersal, the proliferative response was lower than that at the initiation of the study and there were significant differences in the Japanese cedar pollen-specific T-cell response between the start point and 96-week and between 48-week and 96-week $(p=0.022$ and $p=0.045$, respectively). The average SI values of the baseline and 96week intervention were 3.68 and 2.02, respectively. Moreover, there was a significant suppression of the proliferative

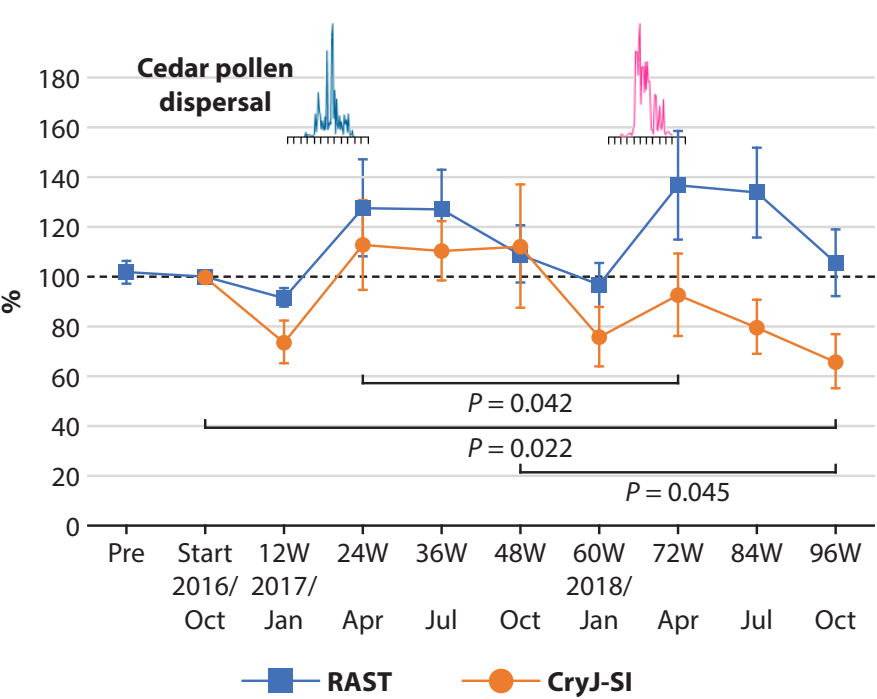

Figure 3. Change in $T$ cell proliferative response to Japanese cedar pollen and levels of serum IgE specific to Japanese cedar pollen in study 2

Each value represents mean \pm SE.

response at 72-week in the second season of the pollen dispersal, compared with that at 24 -week in the first season ( $p$ $=0.042$ ). There was, however, no effect of the transgenic rice on serum specific IgE for Japanese cedar pollen.

\section{Safety profile and tolerability}

Adverse events (AEs) are shown in Table 2. Three patients had mild AEs such as numbness of the left upper limb after blood draw, common cold, and urticaria, however, they promptly recovered and succeeded in taking the test rice. The average intake rate was more than $96 \%$, as shown in Table 3. Three participants dropped out of study 2, two because of pregnancy and one because of a personal reason.

Table 2. Adverse events observed in this study

\begin{tabular}{|l|c|c|c|} 
& Adverse event & Outcome & Causal relationship \\
\hline $20 \mathrm{~g}($ Study 1) & Local pain after blood sampling & Recovery & No \\
Placebo (Study 1) & Common cold-like symptoms & Recovery & No \\
\hline $5 \mathrm{~g}$ (Study 2) & Lower abdominal urticaria & Recovery & No \\
\hline
\end{tabular}

Table 3. Percentage of intake of the tested rice

\begin{tabular}{|l|c|c|c|c|c|}
\multirow{2}{*}{ Group } & \multicolumn{5}{|c|}{ Intake compliance rate (\%) } \\
\cline { 2 - 6 } & 1 12 W & $\mathbf{1 3 ~ 2 4 ~ W}$ & $\mathbf{2 5 ~ 4 8 ~ W}$ & $\mathbf{4 9 ~ 9 6 ~ W ~}$ & Total \\
\hline $\mathbf{5}$ g (Study 1) & 98.3 & 98.7 & & & 98.5 \\
$\mathbf{2 0}$ g (Study 1) & 96.3 & 97.3 & & & 96.8 \\
Placebo (Study 1) & 97.5 & 98.8 & & & 98.2 \\
\hline g (Study 2) & 97.4 & 97.8 & 96.1 & 98.0 & 97.4 \\
\hline
\end{tabular}




\section{Discussion}

In this study we evaluated the efficacy, safety and tolerability of oral intake of 7Crp-expressing rice on patients with JCP. As expected, tolerability was good and no serious AEs occurred, including immediate and late-phase allergic reactions. Although daily intake of $5 \mathrm{~g}$ or $20 \mathrm{~g}$ transgenic rice for 24 weeks did not provide beneficial effects on clinical symptoms and serum IgE levels, long-term intake of $5 \mathrm{~g}$ transgenic rice led to a significant decrease in $\mathrm{T}$ cell proliferative response to Japanese cedar pollen. The reason(s) why 7Crp-expressing rice did not show effectiveness on symptoms remained unclear. It may be due to small sample size or the amount of the test rice. However, the suppressive effect of $5 \mathrm{~g}$ transgenic rice on the $\mathrm{T}$ cell proliferative response in the second disperse season suggests that the intervention period (24-week) was too short to provide the clinical effects.

7Crp used in this study could bind to peripheral blood $\mathrm{T}$ cells in $92 \%$ patients with JCP, however, not to Japanese cedar pollen-specific IgE. ${ }^{8}$ Using T-cell epitope peptide is one of the promising therapeutic strategies; it lacks allergenicity (IgE-binding activity) but retains immunogenicity (T-cell epitopes), and thus, is expected to escape from an IgE-mediated immediate allergic reaction and induce $\mathrm{T}$ cell tolerance. ${ }^{6,7}$ Clinical trials of several T-cell epitope peptides against birch pollen, cats or grass have been performed. Results for some peptides are promising, ${ }^{15-17}$ whereas disadvantages of $\mathrm{T}$ cell epitope peptides include $\mathrm{T}$ cell-mediated late-phase side effects in a considerable number of patients. ${ }^{18}$ Three AEs occurred during our entire study but were transient and patients could continue to consume the test rice. No systemic immediate or late-phase AEs, including gastrointestinal symptoms, were found, indicating the safety profile of the rice.

As subcutaneous or sublingual AIT require several months to years to show its beneficial effects in the treatment of allergic diseases, tolerability is an important issue for these therapies. We selected rice for the delivery of these peptides to patients because of several reasons. ${ }^{9}$ First, rice is a staple food in Japan, and therefore, the $\mathrm{T}$ cell epitope peptide would be easily taken as a daily habit. Second, 7Crp is predominantly accumulated in the protein body I (PB-I), ${ }^{9,10}$ which is resistant to gastrointestinal digestive enzymes. Therefore, the peptide accumulated in the PB-I of rice endosperm is hard to be digested by proteinases, ${ }^{19}$ and thus by itself could be transferred into the tissues of the ileum and jejunum. Indeed, the intact GFP-peptide was detected in the ileum and jejunum in a mouse model when delivered via rice. ${ }^{20}$ Third, the peptide can be expressed in high amounts in transgenic rice seeds (25-62 $\mu \mathrm{g} /$ grain). ${ }^{10}$ In fact, the $5 \mathrm{~g}$ transgenic rice tested in the present study contained approximately $8 \mathrm{mg}$ peptide, as quantified using Western blotting. This dose is very high (approximately ten thousand-fold), compared with the dose of Japanese cedar pollen extract clinically used for subcutaneous or sublingual AIT. Analyses of various animal models indicate that two types of oral tolerance can be induced against antigens; anergy or deletion using high dose of antigens, and regulatory $\mathrm{T}$ cell induction using low dose of antigens. ${ }^{21}$ Moreover, according to the dual-allergen-exposure hypothesis proposed by Lack, ${ }^{22}$ sensitization to food allergens occurs via environmental exposure to the allergen through the skin, and consumption of food allergens induces oral tolerance, on the basis of the close link between eczema and the development of food allergies, suggesting that the oral route is better than the transepidermal route for the induction of tolerance to allergens. These findings indicate that oral administration of 7Crp-expressing rice has potential advantages, compared to conventional subcutaneous or sublingual AIT.

Transgenic rice, which expressed $7 \mathrm{Crp}^{8-10,23}$ two major T-cell epitopes (residues 277-290 of Cry j 1 and residues 246-259 of Cry j 2, ${ }^{24}$ or fragmented and shuffled Cry j 1 and Cry j 2, ${ }^{25-27}$ have shown preventive and therapeutic effects on allergic symptoms and immune reactions to Japanese cedar pollen, resulting in the suppression of specific IgEs in several allergic models. Administration of the peptideexpressing rice before the immunization (preventively) appears to be more effective than that after the immunization (therapeutically). The effects of the peptides on the cytokine profile were reported to be somewhat different. Preventive administration causes suppression of both Th1 cytokines (IFN- $\gamma$ and IL-12p70) and Th2 cytokines (IL-4, IL-5, and IL-10), ${ }^{10,24-26}$ therefore, the primary effect is considered to be due to $\mathrm{T}$ cell anergy or deletion, resulting in the inhibition of differentiation of naïve $\mathrm{T}$ cells into effector $\mathrm{T}$ cells. In contrast, therapeutic administration reportedly leads to the inhibition of Th2 cytokines but not that of Th1 cytokines. ${ }^{23,27}$ These findings indicate that the effects of the peptides are more pronounced on naïve $\mathrm{T}$ cells than those on memory $\mathrm{T}$ cells, and suggest that $\mathrm{T}$ cell-epitope peptide might be suitable for a preventive strategy. The significant result obtained from this study is that successive intake of the $5 \mathrm{~g}$ transgenic rice for 96 weeks led to a reduction in specific $\mathrm{T}$ cell proliferative response to Japanese cedar pollen even during and after the second pollen season. Although the study 2 is an open trial lacking control, it is generally observed that the proliferative response is increased, in association with an increase in specific $\operatorname{IgE}$ titer during and a few months after the pollen season. ${ }^{28-30}$ Since the allergen-specific lymphocyte stimulation test is used to detect the presence of memory $\mathrm{T}$ cells, long-term administration of transgenic rice might also have a significant influence on sensitized memory $\mathrm{T}$ cells. The precise characterization of sensitized memory $\mathrm{T}$ cells is essential to understand how the $\mathrm{T}$ cell epitope peptides influence effector $\mathrm{T}$ cells. Presently, such reduction in specific $\mathrm{T}$ cell response did not induce a decrease in serum IgE level. Further studies are required to elucidate whether decreased $\mathrm{T}$ cell proliferation might provide beneficial effects on symptoms related to JCP and ultimately lead to desensitization. 


\section{Conclusion}

This is the first report evaluating the efficacy, safety and tolerability of the 7Crp-expressing rice in patients with JCP. Owing to limited design of the study, the efficacy of the transgenic rice on allergic symptoms was not recognized by 48 -week trial; however, the decrease in the sensitized $\mathrm{T}$ cell response to the pollen allergen indicates that using peptide-expressing rice is a promising approach and novel form of AIT. Further clinical studies and more precise analyses of immunological effects of ingesting peptideexpressing rice are necessary to overcome JCP.

\section{Acknowledgments}

We thank Siemens AG for measuring allergen-specific $\operatorname{IgE}, \operatorname{IgG}$ and IgG4. The authors declare that no funding was received for the present study. The authors also declare that they have no conflicts of interest. SH, SO, NT, KK, HT, YW, MT, YT and TT designed the study. SH, SO, NT, KK, HT, YW, MT, MT, YT and TT contributed to conception of the study. SH, MK and NM contributed to data collection and performed the statistical analysis. SH and TT wrote the manuscript. All authors read and approved the final manuscript.

\section{References}

1. Holgate ST. The epidemic of allergy and asthma. Nature. 1999;402(6760 Suppl):B2-4.

2. Saito Y. Japanese cedar pollinosis: Discovery, nomenclature, and epidemiological trends. Proc Jpa Acad Ser B Phys Biol Sci. 2014;90: 203-10.

3. Nakamura S, Tsunoda S, Sakaida H, Masuda S, Said AS, Takeuchi K. Analysis of factors associated with cedar pollen sensitization and development of pollinosis in a young Japanese adult population. Allergol Int. 2019;68:39-45.

4. Yamada T, Saito H, Fujieda S. Present state of Japanese cedar pollinosis: the national affliction. J Allergy Clin Immunol. 2014;133:632-9.

5. Masuyama K, Matsuoka T, Kamijo A. Current status of sublingual immunotherapy for allergic rhinitis in Japan. Allergol Int. 2018;67:320-5.

6. Valenta R, Campana R, Focke-Tjkl M, Niedelberger V. Vaccine development for allergen-specific immunotherapy based on recombinant allergens and synthetic allergen peptides: Lessons from the past and novel mechanisms of action for the future. J Allergy Clin Immunol. 2016;137:351-7.

7. Berings M, Karaaslan C, Altunbulakli C, Gevaert P, Akdis M, Bachert C, et al. Advances and highlights in allergen immunotherapy: On the way to sustained clinical and immune tolerance. J Allergy Clin Immunol. 2017;140:1250-67.

8. Hirahara K, Tatsuta T, Takatori T, Ohtsuki M, Kirinaka H, Kawaguchi J, et al. Preclinical evaluation of an immunotherapeutic peptide comprising 7 T-cell determinants of Cry j 1 and Cry j 2, the major Japanese cedar pollen allergens. J Allergy Clin Immunol. 2001;108:94-100.

9. Takaiwa F. Update on the use of transgenic rice seeds in oral immunotherapy. Immunotherapy. 2013;5:301-12.

10. Takagi H, Saito S, Yang L, Nagasaka S, Nishizawa N, Takaiwa F. Oral immunotherapy against a pollen allergy using a seed-based peptide vaccine. Plant Biotechnol J. 2005;3:521-33.

11. Saito S, Hirahara K, Kawaguchi J, Serizawa N, Hino K, Taniguchi Y, et al. Identification of $\mathrm{T}$ cell determinants in Cry J 1 and Cry j 2 of size suitable for immunotherapy against Japanese cedar pollinosis. Annu Rep Sankyo Res Lab. 2000;52:49-58.
12. Goto F, Yoshihara T, Sugimoto N, Toki S, Takaiwa F. Iron fortification of rice seed by the soybean ferritin gene. Nat Biotechnol. 1999;17:182-6.

13. Baba K, Konno A, Takenaka H. Epidemiology. In: Okuda M, editor. Guidelines for Treatment of Nasal Allergy-Perennial Rhinitis and Pollinosis. Tokyo: Life Science Publishing, 2002. p. 8-11. Japanese.

14. Okuda M, Ohkubo K, Goto M, Okamoto Y, Konno A, Baba K. [Japanese allergic rhinitis quality of life questionnaire]. Jpn J Allergol. 2003;52(Suppl 1):21-56. Japanese.

15. Niederberger V, Horak F, Vrtala S, Spitzauer S, Krauth MT, Valent P, et al. Vaccination with genetically engineered allergens prevents progression of allergic disease. Proc Natl Acad Sci U S A. 2004;101(Suppl 2):14677-82.

16. Patel D, Couroux P, Hickey P, Salapatek AM, Laidler P, Larche M, et al. Fel d 1-derived peptide antigen desensitization shows a persistent treatment effect 1 year after the start of dosing: a randomized, placebo-controlled study. J Allergy Clin Immunol. 2013;131:103-9.

17. Ellis AK, Frankish CW, O'Hehir RE, Armstrong K, Steacy L, Larche M, et al. Treatment with grass allergen peptides improves symptoms of grass pollen-induced allergic rhinoconjunctivitis. J Allergy Clin Immunol. 2017;140:489-96

18. Purohit A, Niederberger V, Kronqvist M, Horak F, Gronneberg R, Suck $\mathrm{R}$, et al. Clinical effects of immunotherapy with genetically modified recombinant birch pollen Bet v 1 derivatives. Clin Exp Allergy. 2008; 38:1514-25.

19. Takagi H, Hirose S, Yasuda H, Takaiwa F. Biochemical safety evaluation of transgenic rice seeds expressing $\mathrm{T}$ cell epitopes of Japanese cedar pollen allergy. J Agric Food Chem. 2006;54:9901-5.

20. Wakasa Y, Takagi H, Watanabe N, Kitamura N, Fujiwara Y, Ogo Y, et al. Concentrated protein body product derived from rice endosperm as an oral tolerogen for allergen-specific immunotherapy-A new mucosal vaccine formulation against Japanese cedar pollen allergy. PloS One. 2015;10:e0129209.

21. Weiner HL, da Cunha AP, Quintana F, Wu H. Oral tolerance. Immunol Rev. 2011;241:241-59.

22. Lack G. Epidemiologic risks for food allergy. J Allergy Clin Immunol 2008;121:1331-6.

23. Tsunematsu M, Yamaji T, Kozutsumi D, Murakami R, Kimura S, Kino K. Effect of Cry-consensus peptide, a novel recombinant peptide for immunotherapy of Japanese cedar pollinosis, on an experimental allergic rhinitis model in B10.S mice. Allergol Int. 2007;56:465-72.

24. Takagi H, Hiroi T, Yang L, Tada Y, Yuki Y, Takamura K, et al. A rice-based edible vaccine expressing multiple $\mathrm{T}$ cell epitopes induces oral tolerance for inhibition of Th2-mediated IgE responses. Proc Acad Natl Sci U S A. 2005; 102:17525-30.

25. Wakasa Y, Takagi H, Hirose S, Yang L, Saeki M, Nishimura T, et al. Oral immunotherapy with transgenic rice seed containing destructed Japanese cedar pollen allergens, Cry j 1 and Cry j 2, against Japanese cedar pollinosis. Plant Biotechnol J. 2013;11:66-76.

26. Fukuda K, Ishida W, Harada Y, Wakasa Y, Takagi H, Takaiwa F, et al. Prevention of allergic conjunctivitis in mice by a rice-based edible vaccine containing modified Japanese cedar pollen allergens. $\mathrm{Br} \mathrm{J}$ Ophthalmol. 2015;99:705-9.

27. Fukuda K, Ishida W, Harada Y, Wakasa Y, Takagi H, Takaiwa F, et al. Efficacy of oral immunotherapy with a rise-based edible vaccine containing hypoallergenic Japanese cedar pollen allergens for treatment of established allergic conjunctivitis in mice. Allergol Int. 2018;67:119-23.

28. Wisenbrey $\mathrm{AB}$, Agarwal $\mathrm{MK}$, Offord $\mathrm{KP}$, Adolphson $\mathrm{CR}$, Yunginger JW, Gleich GJ. Seasonal variation of in vitro lymphocyte proliferative response to ragweed antigen E. J Allergy Clin Immunol. 1985;75(1 Pt 1):84-90.

29. Imaoka K, Miyazawa H, Nishimura S, Sakaguchi M, Inouye S. Effect of pollen exposure on serum IgE and IgG antibody responses in Japanese cedar pollinosis patients. Allergol Int. 1996;45:159-62.

30. Moro Y. [Seasonal changes in humoral and cellular immune responses to Japanese cedar (Cryptomeria japonica) pollen allergens in humans with pollinosis]. Jiten. 2003;46:121-33. Japanese. 